\title{
Pedagogic Leadership in Higher Education Using Participatory Action Research Strategies - Illustrative Cases from Three Pedagogical Development Contexts
}

\author{
Sofia Vikstrom ${ }^{1}$, Carina Bostrom ${ }^{1}$ and Annsofi Johannsen ${ }^{2^{*}}$ \\ ${ }^{1}$ Department of Neurobiology, Caring Sciences and Society, Karolinska Institutet, Huddinge, Sweden \\ ${ }^{2}$ Department of Dental Medicine, Division of Periodontology and Dental Hygiene Programme, Karolinska Institutet, Huddinge, Sweden
}

"Corresponding author: Annsofi Johannsen, Associate Professor, Department of Dental Medicine, Division of Periodontology and Dental Hygiene Programme, Karolinska Institutet, Huddinge, Sweden, E-mail: annsofi.johannsen@ki.se

Received date: June 29, 2016; Accepted date: July 22, 2016; Published date: July 29, 2016

Copyright: ( 2016 Vikstrom S et al. This is an open-access article distributed under the terms of the Creative Commons Attribution License, which permits unrestricted use, distribution, and reproduction in any medium, provided the original author and source are credited.

\begin{abstract}
Introduction: In higher education there is a constant need for developments that are sustainable and enhanced quality. The academic leader plays a significant role in that work, which ultimately aims to result in beneficial outcomes for the students. This paper describes how participatory action research as a democratic and inclusive method, can facilitate the academic's participation in their own, as well as in academic programmes' quality enhancement.
\end{abstract}

Objectives: The aim was to illustrate and describe how academic leaders can engage teachers in pedagogical developmental work, such as curriculum development, pedagogic profiles, pedagogical reviews, by using a participatory action research perspective.

Methods: A multiple case study enables the researcher to explore differences within and between cases. In this study, three cases have used a Participatory Action Research model as theoretical frame, to illustrate their quality improvement processes.

Results: All three interventions illustrate improvement processes developed and tested by academic leaders in a university setting, having in common that the teachers involved reflected on the task from their own perspectives.

Conclusion: Our work indicates that these educational approaches have a potential to be of guidance in developing future educational activities, since they spur reflection and participation.

Keywords: Academic leadership; Action research; Education; Development; Implementation

\section{Background}

In higher education there is need for constant developments that is sustainable and enhance quality within the institution, as well as amongst teaching colleagues. Challenges faced by those who hold pedagogical development positions imply a need to put great effort into engaging staff in such pedagogical work. The culture that exists within an educational programme, institution or department has an effect on individual teacher's ability to develop and implement ideas to improve the teaching and learning. Pedagogical leaders commonly need to take a strong socio-cultural perspective in regards to teaching and learning development, and consider the context in which the education takes place. Further, the importance of dialogue in social interaction is described as a crucial element to reach change [1].

\section{Teaching and learning in academic settings}

The idea of developing clear intended learning outcomes for all higher education has been requested by the Bologna Declaration (1999) as well as from government bodies, such as the Swedish Agency for Higher Education. Harden and Crosby [2] argues that teachers must recognize that due to potential information-overload available, students need support in focusing on those learning outcomes necessary for shaping professional practice. The largest driving force to align with the mentioned guiding documents is core curricula, which are developed as an educational programme's highest structure. The most common function to create, adjust and follow-up such curricula's are the pedagogic leaders, such as programme directors or programme board representatives. Their role as managers of education has been presented to entail challenges, such as leading work to develop pedagogical guides and structures. Similarly, Quinn [3] suggested that pedagogical guides should focus on the most important competencies, which the students are expected to achieve.

If the guiding documents are inspired by the above reasoning, students are facilitated to reach the stage of learning, where they change and develop as individuals [4]. This approach might also support students' possibilities to gain crucial practical skills recommended by Schön [5], namely evolving from knowing-in-action to a more conscious approach on that knowledge, called reflection-inaction.

Today's teachers are commonly aware of benefits from reflective learnings activities, for example favouring seminars before multiplechoice exams [6]. Most are also aware of students having different learning-styles and the variation in learning activities that calls for flexible learning methods [7]. Not only do students have different 
learning-styles but teachers also face different teacher roles [2], such as information provider, facilitator, examiner, role-model etc. A distinction often made within university career systems is that own work-related focuses are perceived as constant compromises between producing good research, whilst still having to teach [8]. One promising vein, suggested by Harden and Crosby [2], identified some of the important roles of the medical researcher to relate to pedagogic developments, such as the research-developer and planner roles.

As the workload for teachers not seldom peak at unpredictable intervals, the ambitious inclusive and deep-learning pedagogy suggested beneficial for students, is often challenged, and sometimes have to give way to less ambitious, surface-learning teaching [9]. Stress, high workload and variation in competing work-related tasks increase vulnerability amongst teachers and risks making them return to the traditional knowledge transferring teaching they strive to avoid [10]. As these dilemmas seem to be one that is hard to solve, the suggestion is to identify strategies to facilitate, for those involved, ways to manage coping with them in the most beneficial way possible [11].

\section{Academic leadership}

Empirical studies on academic leadership within the university context has most commonly been focused on heads of universities, whilst limited research has focused on academic pedagogical leaders, such as programme directors responsible for quality and development of an educational programme. The academic leader therefore has a significant role for programme quality, development of teachers within the programme and in the long term learning and teaching outcomes for students [12].

One part of the role as a pedagogical leader has been described to be supportive and coaching amongst teachers in the programme (developer role) but at the same time demanding that they collect student evaluations and produce their feedback and grades on time (deliverer role). These contradictory approaches (support and demand) have been identified to be equally important traits in higher academic leader roles [13]. One of the other main tasks for educational developers has been defined as supporting development of reflective learning amongst teachers [14]. During pedagogical review, the pedagogical leader should highlight for the teacher, be aware of and face the reflection of their teaching in their context, as well as presenting new challenges in teaching. Furthermore, leadership effectiveness in complex environments (such as higher education) requires matching multiplicity in behaviour, including competence in a number of roles and the capacity to move effectively between them [15]. It has been suggested that academic leaders can improve and become more effective in their leadership skills and develop their selfawareness [16]. In a study Vilkinas and Ladyshewsky [17], examined leadership behavior and effectiveness amongst ninety academic Programme Directors (PDs') and found they had the ability to implement and further develop their leadership skills. They also identified PDs had the task of maintaining networks, creating opportunities for changes, and to provide support for teachers.

\section{Participatory action research}

One methodology that has been shown to be beneficiary to reach sustainable change, is a theoretical model of research; Participatory Action Research (PAR). Within the PAR philosophy, important democracy enables and liberates participants to be co-active in constructing deeper understanding with new meaning being underscored. Similar to the PAR, critical theory emphasises change and corresponds well with empowering aspects of quality development. The emancipatory knowledge interest of critical theory is an aspect that fits well within in this multiple case study.

PAR has been suggested to be used as a theoretical frame and structure to empower the academic teacher within the university to engage in the process of constructing and using their own knowledge. This has been shown particularly relevant for people whose role it is to constantly reflect over and evaluate one's teaching and who need to take ownership of existing limitations, and taking initiatives towards desired actions [18]. The PAR philosophy has leant upon above bear common principles, originating from several PAR theorists' work [18].

One of our core assumptions is that pedagogic leaders, in our case within a medical university in Sweden, are drivers in developmental work through a continuous dialogue, enhancing possibilities to reach a sustainable pedagogical change. In the role of the pedagogic leader, it is then important to engage, support and direct teachers by using PAR. In this paper we take a starting point from the subjects of programmes in Occupational therapy and Dental hygiene, as well as in an interprofessional medical masters education. We suggest using a particular PAR model that has shown a promising approach involving teaching researcher (from here on called teacher) in making change, for sustained quality improvement. It is also assumed to beneficially breakdown the polarity between theory and practice [19]. Hence, this paper raises questions on what approaches pedagogic leaders can use to facilitate teaching colleagues to become change agents for the benefit of their student's learning. As there are limited empirical studies focusing on educational quality development taking teachers and their context in consideration, this study focuses on PAR formed, to enhance communication and engagement within different academic teaching cultures.

\section{Aim}

The overall aim was to illustrate and describe how academic leaders in pedagogical developments can engage teachers by using a PAR perspective.

\section{Specific aims:}

- To describe how academic leaders can be engaged in the process of developing a new curricula

- To describe how academic teachers can participate in the process of developing a pedagogic profile

- To describe how academic leaders can support teachers to develop their teaching through a pedagogical performance review

\section{Methods}

A multiple case study [20] enables the researcher to explore differences within and between cases, with a common goal to replicate findings across cases. As comparisons will be drawn, it is suggested to be imperative that the cases are chosen carefully so that the researcher can predict similar results across cases, or predict contrasting results based on a theory [20]. In this study, the three cases were chosen since they illustrated two common assignment pedagogic leaders meeting and one less developed but with a promising teacher development task. In common, the three cases describe a process of change and development, where staff have been included and encouraged to be reflective and critical and thus become drivers of change. Like good teaching, academic leadership is not merely telling or transmitting 
information or ideas; it is a sort of conversation aimed at helping people to change and develop [21]. All three cases have taken inspiration from action research methodology that ultimately aims to engage the group members to be active co-actors in the creation of the new ideas. In particular, John Friedmans Three dimensional leadership model that promote an action-oriented system that is transparent and open to challenge structures and experiment with new ideas to reach sustainable goals [22]. In common for the three cases below is that all processes have included identifying a common goal (or mission) and a more long-term strategy; vision that also include values that ultimately aim to support students' team competence.

Also, in common when planning the improvement process for developing new curricula, a pedagogic profile or a pedagogic performance interview our strategy was to use a small group collaborative set-up [23] to encourage participants to become owners and drivers of the quality improvement processes performed.

\section{Theoretical framework}

The theoretical model of research (Participatory Action Research; PAR) constitutes the foundation within which the three following implementation processes are tested and described. Although, implementations differ in focus, they all apply PAR as a theoretical method and frame to empower teaching colleagues within the university to engage in the process of building and using their own knowledge. Here, as in the PAR philosophy, setting a structure that enables and liberates participants to be co-active in constructing new meaning, through dialogue or group discussions, are underscored, independent of whether they constitute a process of dialogue or discussions in smaller or larger groups [24].

Based on the belief that PAR can lead to sustainable quality improvements that ultimately benefit the students' as well as their teachers' development over time, the academic developments presented are framed within a specific quality development model encompassing four phases; plan (P), do (D), study (S) and act (A) (commonly known as the PDSA-cycle [24] (Figure 1). This model works particularly well within a system or organisational structure of interrelated processes operating to produce an output, such as a university, and works well alongside the quality improvement viewpoint, which involves understanding how the system works and shares that knowledge within the organisation [25]. The PDSA cycle may comprise of interactions involving mental activity, attitudes, and behaviours of a single human or amongst a group of people orchestrated to produce services and outcomes. The goal of PDSA quality improvement is to pursue effective changes in processes that favourably affect outcomes [24].

PDSA cycles form part of a concept called "The Improvement Guide" [26], which provides a framework for developing, testing and implementing changes leading to improvement. The model is based in scientific method and moderates the impulse to take immediate action with the wisdom of careful study. The framework includes three key questions and a process for testing change ideas [26], for the overarching drivers of the larger set-up and evaluation. The three key questions are: i) what are we trying to accomplish? ii) How will we know that a change is an improvement? And iii) what changes can result in improvement? The three improvement processes described in the findings section below have in common that they have all applied the circular part of the PDSA improvement model on their work. However, they have reached different stages. The three case-reports on initiatives taken to facilitate pedagogical developments are presented and problematized from the challenges of versatile demands faced by teachers within higher education. They are further discussed from a multiple case perspective [20] as well as from a current health care practice trend-perspective.

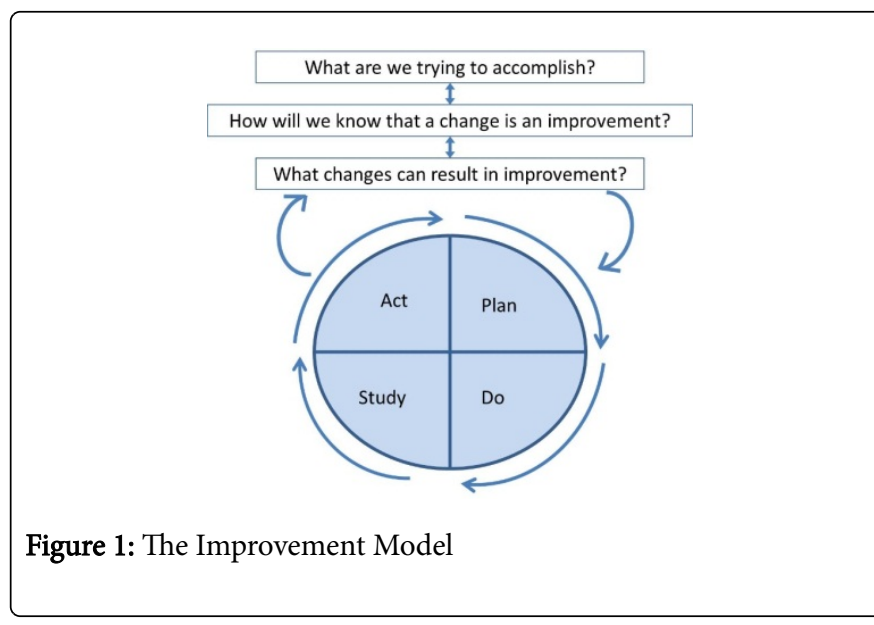

\section{Findings}

The structure for this work lean on the PDSA model of improvement by Langley et al. [24], the three cases address the following aspects of pedagogic developmental work using PAR and PDSA-model:

\section{- Curriculum developmental work}

- Implementation of a pedagogical profile

- Pedagogical performance review

The three improvement processes described in the findings section below have incommon that they have all applied the circular part of the PDSA improvement model on their work.

\section{Curriculum Developmental Work}

This case aimed at renewing a bachelor programme curriculum. A multi-faceted challenge that provided reason for incorporating all faculty members and clinical teachers involved in the programme.

\section{The curriculum development process}

This development process took place in collaboration with teachers and students within an occupational therapy programme.

Plan: The initial step of establishing the project's status was done by asking the teacher groups to discuss and identify a common direction that all agreed upon. The pedagogic leader took initiatives to display future challenges, from different futuristic reports to view the need for change. This was discussed and problematized in relation to the current programme.

Do: The pedagogic leader formulated the needs and goals in specific areas. The colleagues participated in identifying subjects that would work to answer the question of "How to secure improvement" in the particular think-tank subject area. The think tank areas focused on; inter professional learning and clinical practice; the main subject and aspects within the programme; aspects of recruitment of students and its profile; entailing both generic competences and pedagogical profile. 
The think-tanks were based on voluntary choice and participation was strongly advised.

Study: The pedagogic structure applied by all think-tank members was to search for and contribute by having read a text they had brought and reflected around for the group to discuss. The pedagogical leader encouraged the think-tank members to engage and reflect on different data collected such as pedagogical publications and national guides. Thereafter, summarizing all suggestions for change identified by the group in a text that would also form parts of the text in the new draft curricula.

\section{Reflection of curriculum development}

Weaknesses in the set-up concern the time and multiple competing tasks that teachers were facing. Strengths were that the teachers, feel more like co-creators and co-owners of the final product; the curricula. The pedagogic leaders had thus made it more appealing through allowing choice of subject. This corresponded with the thoughts presented by Kolb and Kolb [7], who suggested creating a structure that enabled space for mutual experiential learning, simultaneously corresponding with each person's preferences.

\section{Implementation of a Pedagogical Profile}

\section{Development of the pedagogical profile}

This implementation work is informed by teachers from different subject-orientations within a multiprofessional programme.

Together with the programme committee chair person, a group of teachers from a multi-professional programme committee created a draft of a pedagogical profile of the courses included in the programme committee. The profile was about teaching that aimed for the students to reach a number of general skills following training (evidence-based approach, readiness to act, information literacy, communication skills, multi-perspective viewing, www.ki.se).

\section{Implementing the pedagogical profile}

Plan: Then, course responsible teachers in the programmes were invited to try to adapt their course curricula to the profile, and some core concepts were introduced. To further be able to implement the profile based on the course responsible teacher's experiences, another workshop was held.

Do: Before the workshop, each participating teacher read one or two articles about competence and performed written reflections in the form of a reading log. The teachers took the reading log to the workshop and discuss their reflections. Prior to the workshop, teachers also analyze current course curricula in relation to one or two competences in the pedagogical profile. The aim of the workshop and implementation is to support the course responsible teachers to change their course curricula so that they are in line with the pedagogical profile. In addition, it aimed to enhance skills applicable in formulating learning objectives, learning activities, grading criteria and grading in line with the pedagogical profile.

Study: Teachers are supported in the implementation process by a group of teachers responsible for implementation but especially by one another, via implementation specific activities in the university educational website. Here, each teacher will be asked to enter how they implemented the profile in the form of teacher activity, examinations, grading criteria and learning outcomes. The idea is for teachers to use good examples to find their own ways to implement the pedagogical profile based on a PAR-perspective, namely that teachers together are responsible for the implementation. During the implementation period teachers communicate with other teachers within the educational webplatform used. A final workshop was held where following-up on the exercises that the teachers performed concerning implementation of the pedagogical profile.

\section{Evaluation of the pedagogic profile}

Evaluation of the implementation of the profile was performed by gathering students 'and teachers' views on how the profile attained the potential need for changes in the profile and the benefits yielded by it and how it has been implemented. Heads of department were involved in suggesting which teachers would be involved, increasing the chances that the teacher will get time for participation in the implementation process.

\section{Pedagogical Performance Review}

This example originates from individual dialogues with teachers within a Dental hygiene programme. The teachers in an educational programme have regular performance reviews with the programme leader to discuss different issues in general. In this case, a pedagogic performance review will be highlighted and has been developed and implemented in the educational activities amongst teachers, to support pedagogical issues, activities and providing space for reflection and creativity. This case will also focus on the leadership capabilities and the leader's role in higher education.

\section{Development of the performance review}

Plan: The initial step was to reflect what pedagogical performances should include teachers and what these dialogues with teachers would focus to accomplish in their teaching skills. The next step was to construct this pedagogical performance review, inspired by a Medical Education guide 5 [6] and the Karolinska Institutet model for supervisor review [6]. The performance interview guide entailed open questions and the pedagogical review was divided into different themes; formal competence, approach and experience of teaching, development of teaching materials, reflection of pedagogical issues, interaction with other teachers in other organisations, and short-term, as well as long term goals, with teaching and learning.

Do: Before the pedagogical performance review was implemented, the involved teachers were contacted and informed of the purpose of the interview, thus giving them time to prepare and reflect. During the interview, questions were asked in order to highlight the themes above. More specific questions, regarding for example how the teachers plan and work with their teaching in relation to learning outcomes, their learning activities and examinations, their opportunity to develop pedagogy in their teaching, their work with student's participation in the teaching, and reflection on their educational awareness. The teachers should also think about how the changes are an improvement in their teaching.

Study: The outcome of the pedagogical performance review was evaluated three months after the interview using a questionnaire, where the interviewees were asked if their expectations were fulfilled. Questions also included their opinion of the importance of this type of dialogue as an instrument for quality management. As an academic leader of an educational programme, working with pedagogical review 
with employees, aims at enabling and making improvements visible both in the activity, by teachers themselves, together with colleagues and ultimately for the students, where the goal is to improve teaching and learning. In learning organizations the goal is to achieve a "shared vision" amongst each other and an environment of creativity and enthusiasm. This pedagogical performance review could work well as a strategy to highlight the pedagogical activities, provide space for reflection and creativity.

\section{Reflection of the pedagogical performance review}

The implementation of the pedagogical performance review was introduced in order to visualize the educational activities and strengthen the educational culture in the organisation. All teachers that participated was very positive to this interviews, maybe one possible hindrance to use performance review might be that not all teachers are equally interested in these specific issues. The pedagogic leaders have an important role to support and inspired the teachers for example develop pedagogical issues.

The role of the pedagogic leaders, in different stages of the PDSA model, as they have been used in the projects of the study:

Plan: As shown above, the general direction of the teacher and learning activity was set in this planning stage. Here the pedagogical leaders play a big part in setting the agenda and delivering ideas on how to proceed and points towards a long term goal, which in our case, was to implement a pedagogical profile; a curricula and a pedagogical performance interview routine. The main goal of the pedagogic leaders for the three cases presented in this paper was to try to reach sustainable change by the mutual use of pedagogics.

Do: In the doing phase, the three pedagogic leaders (and authors) encouraged colleagues to reflect on their current state of performing teaching and learning. This way they tried to set the scene so that existing flaws in the educational setting were highlighted and recognized.

Study: In the study phase, the pedagogic leaders engaged staff discussions on how to secure a change that was in fact an improvement. Also, they needed to secure the necessity of the change to be well-founded and internalized in all participants. For example, the pedagogic structure applied in the think-tanks used in the curricula development work, was a modified form of creating the work as a joint enterprise concerning teaching and learning [27], where all teaching staff were drivers of change who contributed and reflected on improvements needed.

Act: In the act-phase, suggestions for developments were verbalised and documented, and thus made an important foundation for future follow-ups. The pedagogical leaders not only assisted in formulating these actions but also encouraged and showed direction in ways to follow them through. In the pedagogical review example, the leaders encouraged colleagues to pursue the goals set at the meeting and made sure to have regular continued meetings.

\section{Discussion}

\section{Main findings}

The three examples of pedagogic development illustrated in this multiple case study [20] are set up, ultimately to enable students to gain competencies to meet the constantly changing demands from health care [28]. Through implementing more interactive teaching strategies and internalizing structures for pedagogic development and agency, students are more likely to gain increased skills of use in their future work as reflective practitioners [5,29], and reach the general competencies and specific learning outcomes stated in e g. curricula's and profiles. This has been shown by Capobianco [30], whose results from action research strategies to develop the education to become more gender neutral indicated that teachers as researchers of their own practice gained new knowledge about teaching and generated a cluster of pedagogical possibilities for inclusive, dynamic teaching.

\section{Comparison between the cases}

On an individual note: One beneficial starting point in this developmental work was that all participating teachers already possessed collaborative learning competencies which are important since they lay a beneficial foundation for being open to change and willingness for being flexible. Also, mutual insights by all staff, in the learnings outcomes, as well as, the goals of professional competencies are a prerequisite for implementation and regular use [31]. These include cooperative teaching skills, like being on time, showing a balance between active and passive listening and participation. They also pose usable and reflective questions, and share information and personal understanding. Through individual studies and personal experiences, they have appropriate knowledge and the ability to explain it to others, which also illuminate social competence and a familiarity with giving and receiving feedback [31].

Our findings also illustrate how the use of the teachers' involvement and contributions in developing the programmes contribute to a social action process where all cognitive resources and ideas are taken into account [31]. On an individual note, suggestions are that descriptions and documentation of one's own educational work could stimulate reflection and promote professional development of academic teachers, which might also generate scientific publications on pedagogical development that can raise the academic renomeé from a pedagogical viewpoint [32]. Thus, using the combination of case study methodology and action research approaches provide an excellent insight and learning activity for the scholars and the pedagogic leader her/himself.

From a group perspective: In terms of students learning, some trends indicate the values of group-work and case-based educations and letting students study beforehand whilst using teachers mainly in as facilitators in seminars (flipping the classroom) [33]. These strategies allow more interactive teaching and have been shown to enhance learning [34]. In addition, learning is to be best performed through collaborations. The most common definition of 'collaborative learning' is that it is a situation in which two or more people learn or attempt to learn something together.

Organisational aspects: Collaborating and negotiating with others has also been shown particularly relevant for people whose role it is to constantly reflect over and evaluate one's teaching. Commonly university teachers, face having to take ownership of existing limitations and take initiatives towards desired actions [18]. In our views, the final goal achievement is not to get the action "right" or even to maximize effectiveness within an existing conceptual framework, or in McRoy and Gibbs [13] terms "only take on a deliverer role" as academic leaders. Rather, it is to move on a collective journey towards a new understanding of what we are doing, which in itself would open up new possibilities for action as joint reflective practitioners [5] with sense of pedagogical agency [29]. 
We also believe it is important to use developmental models and strategies concerning pedagogical issues and to make improvements visible and thereby increase teacher's self-esteem. It is also central to perform pedagogical activities amongst teachers and within the organisation, such as how to work, attitudes and to motivate each other and develop pedagogical issues, thereby maintaining high quality standards of teaching and learning $[27,35]$.

\section{Pedagogical implications}

One benefit of initiating dialogue-based educational development is to gain an understanding and knowledge of teachers' attitude towards education; their ideas about learning and teaching; their learning activities in teaching and their approach to teaching. All these elements are significant key elements for the quality of education. It is also important for teachers to become more aware of their own approach to teaching and learning, which our work made feasible. Trigwell et al. [36] suggested that there is an association between one's approach to knowledge and awareness of one's knowledge, which contributes to how students learn. Our findings match research stating the importance to provide regular opportunities to develop the organization's human capital, allowing people to develop knowledge and skills that they can put to good use in their different contexts [37]. Furthermore, surpluses that teachers feel are that their efforts bear some fruit, thereby contributing to a sense of efficacy that is vital to future improvements. This study also supports the value of higher education and pedagogical leaders to reflect in their organisational work from a variety of perspectives. There is a need to be aware that the organisations are often scarce in resources, where differences in needs, interests, perspectives, power, and influence constantly affect the work of both academic leaders and teachers [37]. Similarly, Lieff and Albert [38] stated that complex issues, such as resource personnel, time, facilities, or finance, were believed to be behind some of the tensions in educational work. The findings, correspond with Quinn [3], illustrating that pedagogical leaders benefit from setting and following an agenda, map the distribution of power and interests, develop strategy, build linkages, and negotiate. In universities where time is of the essence, we suggest employing management as well as pedagogical leaders encourage teaching staff to enhance their pedagogical competencies. As mentioned earlier, Harden et al. [2] identifies this as the pedagogical challenges of medical researchers; to also address the developer role in relation to educational tasks. If such roles are ignored, or under-acknowledged it is suggested to threaten inclusive, deep-learning pedagogics [9].

Hence, universities need pedagogical leaders who have a mandate to act in defense of pedagogical educations and skills so that the research departments and divisions influence on educational programmes are weighted against quality of teaching and learning [39]. It is also important to have pedagogical leader who take responsibility in highlighting pedagogical and educational needs and limitations. If their work and competencies are promoted and used wisely over a prolonged period of time there are likely to be large benefits for students' learning.

\section{Methodological considerations}

On the basis of the above, our display of PAR usage as a frame work for quality improvement provides good possibilities for transferability. Further, it is relevant to anyone involved in the pursuit of health care education generally and teacher education and development particularly.
A critique to previous PAR implementations is that they often either focus on change or on quality improvement, where few have combined the two into a joint venture of change and quality development [25]. We argue that our implementations opt for change that, in turn, will have a likely positive effect on the perceived quality in the education of students. Mutual for the three cases is that a pedagogic vision / profile is either developed by, or introduced to, the staff and that the pedagogical leaders have not only been deliverers of teaching and learning strategies in a pedagogical structure that is pre-decided and fixed but have taken on a developer role. In correspondence with McRoy and Gibbs [13] we also found that academic leaders reach good progress in quality development by planning and then inviting teaching colleagues to co-act in describing, developing and using a future course of direction.

\section{Conclusion}

The study illustrates PAR as a potential strategy to highlight educational activities, provide space for reflection and creativity on the individual and at the organizational level, as well as an overview of how the system enables or hinders the quality of education.

We have illustrated and described how academic leaders in pedagogical developments can engage teachers by using a PAR perspective, and it seems to be a fruitful method to use when initiating and performing pedagogical development and change within a medical university setting.

Here, taking use of the pedagogic leaders developer roles have shown highly important indicating that they have the potential not only to be used as deliverers of new directives, structures and ideas, but also be able to be developers of the pedagogic work within the organization, and between colleagues who contributes to education.

\section{References}

1. Barman L, Laksov KB, Silén C (2014) Policy enacted - teachers' approaches to an outcome-based framework for course design. Teach in Higher Educ 19: 735-746.

2. Harden RM, Crosby J (2000) AMEE Guide No 20: The good teacher is more than a Lecturer - the twelve roles of the teacher. Medical Teacher 22: 4.

3. Quinn RE, Faerman SR, Thompson MP, McGrath MR (1996) Becoming a master manager: a competency framework, 2nd edn, New York, Wiley pp: 9-24.

4. Marton F, Dall'Alba G, Beaty E (1993) Conceptions of learning. Int J of Educ Rese 19: 277-300.

5. Schön D (1983) The Reflective Practitioner: How Professionals Think in Action Donald A Schon: Basic Books.

6. Barman L, Sandberg AK, Weurlander M (2010) Guide for pedagogical merits. CME-guide no 5, Karolinska Institutet.

7. Kolb AT, Kolb DA (2005) Learning styles and learnings spaces: Enhancing experiential learning in higher education. Academy of Manage Learn and Educ 4: 193-212.

8. Kember D (1997) A reconceptualization of the research into academics conceptions of teaching. Learning \& Instructions 7: 255-275.

9. Fraser SW, Greenhalgh T (2001) Coping with complexity: educating for capability. BMJ 323: 799-803.

10. Biggs J, Tang C (2007) Teaching for Quality Learning at University Maidenhead: Open University Press/McGraw Hill.

11. Askling B, Stensaker B (2002) Academic leadership: Prescriptions, practices and paradoxes. Tertiary Educ and Manage 8: 113-125.

12. Briggs ARJ (2001) Academic middle managers in further education: reflections on leadership. Res in Post-Compulsory Educ 6: 223-237. 
Citation: Vikstrom S, Bostrom C, Johannsen A (2016) Pedagogic Leadership in Higher Education Using Participatory Action Research Strategies - Illustrative Cases from Three Pedagogical Development Contexts. Dentistry 6: 388. doi:10.4172/2161-1122.1000388

Page 7 of 7

13. McRoy I, Gibbs P (2009) Leading change in higher education. Educ Manage Admin \& Leadership 37: 687-704.

14. Land R (2001) Agency, context and change in academic development. Int J for Academic Development 6: 2-20.

15. Denison DR, Hooijberg R, Quinn RE (1995) Paradox and performance: toward a theory of behavioral complexity in managerial leadership. Org Sci 6: 524-540.

16. Vilkinas T, Shen J, Cartan G (2009) Predictors of leadership effectiveness for Chinese managers. Leadership \& Org Development J 30: 577-590.

17. Vilkinas T, Ladyshewsky RK (2011) Leadership Behaviour and Effectiveness of Academic Program Directors in Australian Universities. Educa Manage Admin \& Leadership 40: 109-126.

18. Koch T, Selim P, Kralik D (2002) Enhancing lives through the development of a community-based participatory action research programme. J Clin Nurs 11: 109-117.

19. Kemmis S, McTaggert R (1988) Introduction: The nature of action research. In Action Research Planner (3rd ed) (Kemmis S \& McTaggart R eds.), Deakin University Press, Victoria pp: 5-28.

20. Yin RK (2003) Case study research: Design and methods (3rd ed.) Thousand Oaks, CA: Sage.

21. Ramsden P (1998) Learning to lead in higher education. London: Routledge.

22. Friedman J (2011) Actions-Influence-Expertise: A Planning Tool Published by PR News Press. PR News Corporate Social Responsibility \& Green PR Guidebook 5: 12-14.

23. Wheelan S (2009) Group size, group development and group productivity. Small Group Res 40: 247-266.

24. Langley GL, Nolan KM, Nolan TW, Norman CL, Provost LP (2009) The Improvement Guide: A Practical Approach to Enhancing Organizational Performance (2nd Edition) Jossey Bass, San Francisco.

25. Speroff T, O'Connor GT (2004) Study designs for PDSA quality improvement research. Qual Manag Health Care 13: 17-32.

26. NHS (2014) Quality and service improvement tools - PDSA (Plan, Do Study, Act).

27. Laksov KB, Mann S, Dahlgren O (2008) Developing a community of practice around teaching: a case study. Higher Educ Res and Development 27: 121-132.
28. Frenk J, Chen L, Bhutta ZA, Cohen J, Crisp N, et al. (2010) Health professionals for a new century: transforming education to strengthen health systems in an interdependent world. Lancet 376: 1923-1958.

29. Kahn P (2009) Contexts for teaching and the exercise of agency in earlycareer academics: perspectives from realist social theory. Int $\mathrm{J}$ for Academic Development 14: 197-207.

30. Capobianco BM (2007) Science Teachers' Attempts at Integrating Feminist Pedagogy through Collaborative Action Research. J of Res Sci Teach 44: 1-32.

31. Hedin A (2006) Learning at high-level. Division for Educational Development, Uppsala University, Sweden. University Press, Uppsala pp 279-307.

32. Tigelaar DE, Dolmans DH, de Grave WS, Wolfhagen $\mathrm{IH}$, van der Vleuten CP (2006) Portfolio as a tool to stimulate teachers' reflections. Med Teach 28: 277-282.

33. Deslauriers L, Schelew E, Wieman C (2011) Improved learning in a largeenrollment physics class. Science 332: 862-864.

34. Crouch CH, Mazur E (2001) Peer instruction: Ten years of experience and results. Am J Physics 69: 970.

35. Roxå T, Mårtensson M, Alveteg M (2011) Understanding and influencing teaching and learning cultures at university: a network approach. Higher Educ 62: 99-111.

36. Trigwell K, Prosser M, Waterhouse F (1999) Relations between teachers' approaches to teaching and students' approaches to learning. Higher Educ 37: 57-70.

37. Gunderman RB, Chan S (2007) Where is the action in organizational learning? Radiology 242: 650-653.

38. Lieff SJ, Albert M (2010) The mindsets of medical education leaders: how do they conceive of their work? Acad Med 85: 57-62.

39. Trowler P, Ali C (2002) Teaching and Learning Regimes: Implicit theories and recurrent practices in the enhancement of teaching and learning through educational development programmes. Higher Educ Res \& Development 21: 221-240. 